Rapid Communications

\title{
Microplate-based Assay for Screening of Advanced Glycation End Products Binding to Its Receptor
}

\author{
Deepak Ganesh,* Kyoko Torigoe,** Miyuki Kumano-KuramochI,** Sachiko MachIDA,** and \\ Toshiro KOBORI $*, * * \dagger$ \\ *Graduate School of Life and Environmental Sciences, University of Tsukuba, 1-1-1 Tennodai, Tsukuba, \\ Ibaraki 305-8577, Japan \\ **Food Research Institute, National Agriculture and Food Research Organization, 2-1-12 Kan-nondai, Tsukuba, \\ Ibaraki 305-8642, Japan
}

\begin{abstract}
Advanced Glycation End products (AGEs) are a group of amino-acid modifications produced with sugars or di-carbonyls. Some AGEs are known to affect health through binding to the receptor of AGEs (RAGE). Here, we propose a method for screening RAGE-binding AGEs by a competitive assay using purified RAGE and AGEs-specific antibody. This method has clarified that at least carboxyethyl lysine and pentosidine among methylglyoxal-derived AGEs are involved in RAGE binding, suggesting that this would be a promising method for classifying RAGE-binding AGEs.
\end{abstract}

Keywords Advanced Glycation End products (AGEs), Receptor for Advanced Glycation End products (RAGE), carboxyethyl lysine, pentosidine, lysine methylation

(Received December 25, 2018; Accepted December 28, 2018; Advance Publication Released Online by J-STAGE January 11, 2019)

Advanced glycation end products (AGEs) are formed by modification of amine groups in the N-terminal of proteins or side chains of lysine and arginine residues through a nonenzymatic Maillard reaction with reducing sugars and dicarbonyl compounds. ${ }^{1}$ AGEs are produced in the cooking process, and play important roles in taste, flavor, and color of processed food. ${ }^{2}$ On the other hand, it has also been found that AGEs, which are endogenously formed with metabolites in the body, are involved in various health effects, such as diabetic complications. ${ }^{3}$ Therefore, effects of dietary and endogenous AGEs on health have been of great interest. ${ }^{4}$

The receptor for AGEs (RAGE) is a member of immunoglobulin superfamily of cell surface receptors. ${ }^{5}$ RAGE is a patternrecognition receptor consisting of an extracellular region with $\mathrm{V}$-, C1-, and C2-domains, a transmembrane domain, and a short tail.6,7 V- and C1-domains are primary interacting sites for various ligands including AGEs as well as non-AGEs ligands, such as high-mobility group protein box- $1^{8}$ and amyloid- $\beta$ protein. ${ }^{9}$ The tail region takes part in signal transduction triggered upon ligand binding to RAGE. ${ }^{6,7,10}$ Therefore, it is significant to classify RAGE-binding AGEs as a stimulating factor for medical applications and improved food processing.

A single sugar or di-carbonyl compound together with a protein produces multiple chemical modifications as AGEs, and growing numbers of chemical species have found to be AGEs so far. ${ }^{1}$ Individual AGEs possess specific biological and chemical properties. For examples, AGEs do not always bind to RAGE, as observed in carboxymethyl lysine, ${ }^{11}$ and different types of AGEs produced in a single protein are thought to be concertedly involved in RAGE binding. Moreover, there is a risk that some

$\dagger$ To whom correspondence should be addressed.

E-mail: tkobo@affrc.go.jp
AGEs would be degraded upon acid hydrolysis required for conventional amino acid analysis by HPLC. These natures of RAGE and AGEs have made it difficult to clarify the involvement of individual AGEs in binding to RAGE.

We have succeeded in the overexpression of extracellular region of human RAGE (soluble RAGE; sRAGE) as a biotintagged form in silkworm; purified sRAGE stably maintains the AGEs binding activity. ${ }^{12}$ Hereby using this biotin-tagged sRAGE, we have proposed a method for screening and identifying individual AGEs on a protein in binding to RAGE. Although this method is primarily based on a 96-well plate assay using sRAGE with an AGEs-specific antibody as a competitor (Fig. 1), a bottleneck of this method is the limited availability of an antibody. To reduce this technical limitation, therefore, a lysine methylation step was optionally introduced prior to the assay. Namely, first, AGEs are formed on a protein with or without lysine methylation, thus allowing a brief screening of lysine- or arginine-derived AGEs contributing to RAGE binding. Second, AGEs on native and methylated proteins are applied to a competitive assay where sRAGE as a sensing molecule is together with an AGEs-specific antibody as a competitor.

In this study, AGEs formed on bovine serum albumin (BSA) by methylglyoxal (MGO) were taken as a model, since MGO is found under dietary and endogenous conditions. After preparation of lysine-methylated BSA (MeBSA), as described previously, ${ }^{13}$ AGEs were produced on BSA and MeBSA through incubation with various concentrations of $\mathrm{MGO}$ at $37^{\circ} \mathrm{C}$ for 1 week. $^{14}$ To characterize these samples, the modification ratios of lysine and arginine residues after MGO treatment were evaluated by fluorescamine and 9,10-phenanthraquinone, respectively. ${ }^{15,16}$ In the case of MeBSA, it was found that approximately $80 \%$ of the lysine residues were methylated 
A

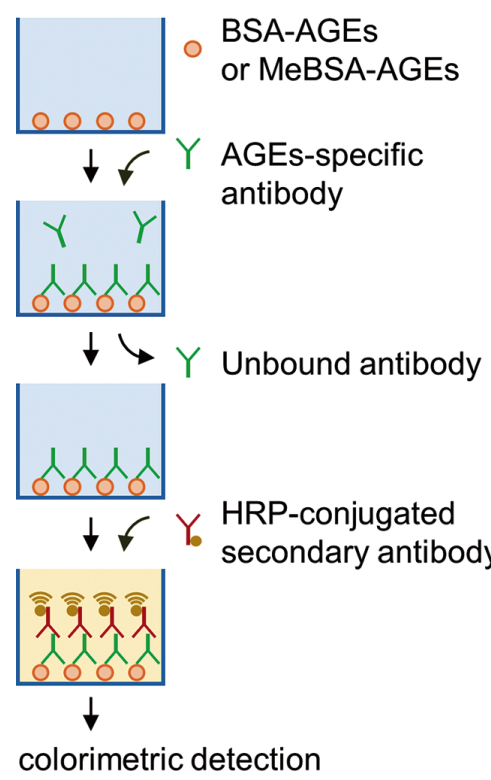

B

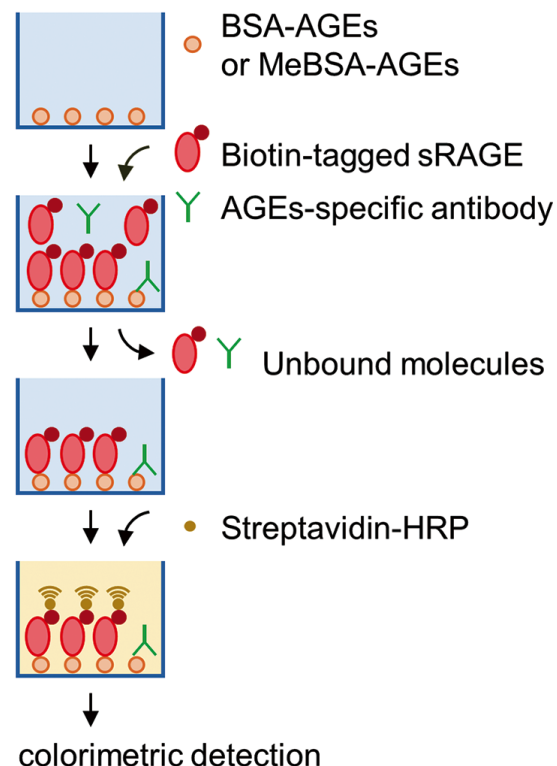

Fig. 1 Scheme for the screening of AGEs binding to sRAGE. Standard direct ELISA using AGEsspecific antibody was used to identify AGEs produced on BSA upon MGO treatment (A). Competitive assay using biotin-tagged sRAGE and AGEs-specific antibody was applied to identify RAGE-binding AGEs (B).

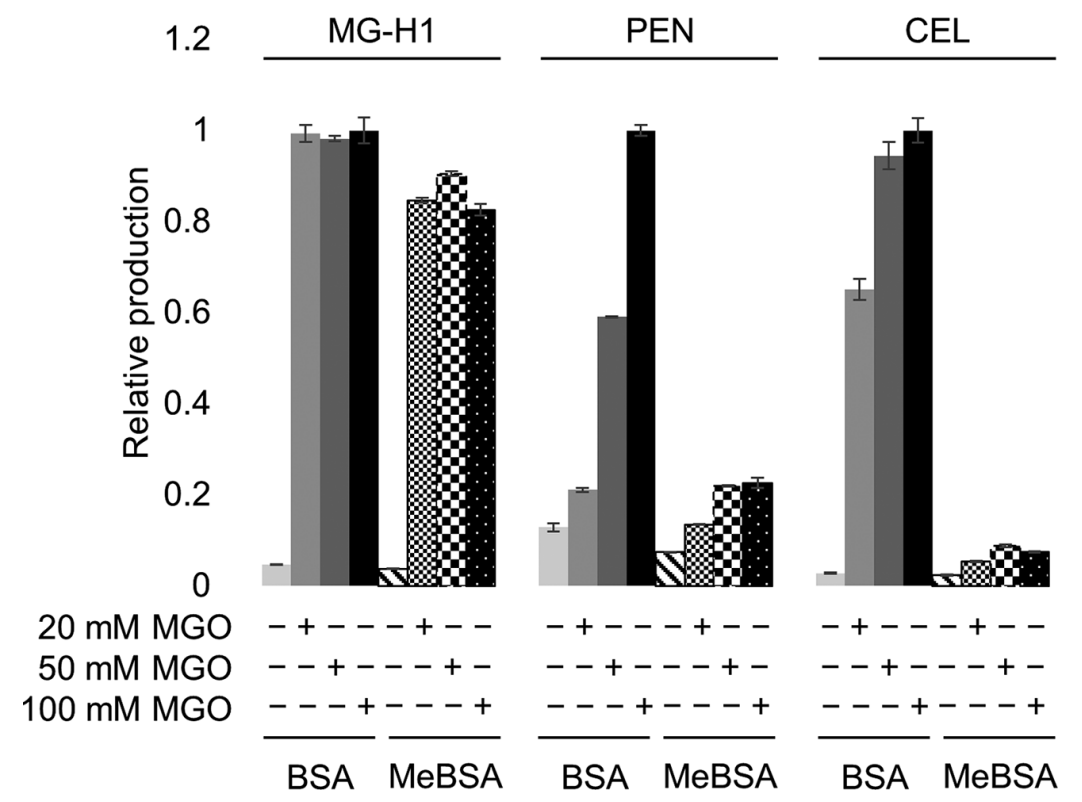

Fig. 2 Production of MG-H1, PEN and CEL on BSA-AGEs and MeBSA-AGEs analyzed by direct ELISA. Antibody binding to each sample was normalized based on the absorbance measured in BSAAGEs prepared by $100 \mathrm{mM}$ MGO. Monoclonal antibodies used were as follows: anti-MG-H1 Ab (Hycult Biotech); anti-PEN Ab (Trans Genic); anti-CEL Ab (Trans Genic); HRP-conjugated antimouse IgG Ab (BioRad).

(Fig. S1A in Supporting Information). The ratio of the modified lysine residues was constantly $\sim 80 \%$ in MeBSA treated with 20 , 50 , and $100 \mathrm{mM}$ MGO, suggesting that the lysine residues mostly protected any successive reaction by MGO. In the case of BSA treated with 20, 50, and $100 \mathrm{mM} \mathrm{MGO,} \mathrm{lysine}$ modification was promoted upon increasing MGO concentration (Fig. S1A). On the other hand, profiles of arginine modification ratios of BSA-AGEs and MeBSA-AGEs apparently overlapped each other (Fig. S1B in Supporting Information), and $60-70 \%$ of arginine residues were constantly modified as AGEs in BSA as well as MeBSA upon MGO treatment. These results suggest that the methylation of lysine prior to MGO treatment would not largely interfere with arginine modification in MeBSA-AGEs.

The production levels of methylglyoxal-derived-hydro- 
imidazolone (MG-H1), pentosidine (PEN) and carboxyethyl lysine (CEL), which are known to be major AGEs produced by MGO, ${ }^{17}$ were estimated by standard direct ELISA using antibodies specific to these AGEs (Fig. 1A and Fig. 2). Although the production level of MG-H1 among MeBSA-AGEs was lower than that among BSA-AGEs, MG-H1 was constantly produced regardless of the MGO concentration. This suggests that MG-H1 would be fully saturated on arginine residues in BSA and MeBSA upon at least $20 \mathrm{mM}$ MGO treatment. On the other hand, the productions of PEN and CEL among samples of BSA-AGEs were increased depending on the MGO concentration. The production levels of PEN and CEL were also found to be significantly lower in samples of MeBSA-AGEs compared to samples of BSA-AGEs. This observation could also be attributed to the methylation of lysine residues in MeBSA, since lysine residues were required for CEL and PEN production.

The bindings of sRAGE with BSA-AGEs and MeBSA-AGEs absorbed on the wells were analyzed using purified sRAGE (Fig. 1B). Significant binding was clearly observed only when BSA-AGEs prepared with 50 and $100 \mathrm{mM}$ MGO were used for absorption (Fig. 3A). The binding profiles of these AGEs with sRAGE exhibited a plateau at their concentrations of more than $10^{-2} \mathrm{~g} / \mathrm{L}$. On the contrary, in the case of BSA-AGEs prepared with $20 \mathrm{mM}$ MGO, sRAGE binding was totally suppressed, even at concentrations of $10^{-2} \mathrm{~g} / \mathrm{L}$ or more. On the other hand, sRAGE did not bind to MeBSA-AGEs, regardless of the MGO concentration (Fig. 3B), suggesting that AGEs possibly produced on arginine with MGO, including MG-H1, argpyrimidine and tetrahydropyrimidine, ${ }^{18}$ would not largely contribute to sRAGE binding.

To assess any effect of AGEs produced on BSA on sRAGE binding, a competitive assay using sRAGE and an AGEsspecific antibody was performed (Fig. 1B and Fig. 3C). When BSA-AGEs prepared with $100 \mathrm{mM}$ MGO was used as a substrate for the assay, the maximum sRAGE binding to this BSA-AGEs was observed in the absence of any antibody in the reaction mixture. When anti-MG-H1 Ab was added to the wells prior to sRAGE addition, no detectable change in the sRAGE binding to BSA-AGEs was observed, suggesting that MG-H1 would not interfere with sRAGE binding to BSA-AGEs. On the contrary, anti-PEN $\mathrm{Ab}$ and anti-CEL $\mathrm{Ab}$ inhibited sRAGE binding to BSA-AGEs, suggesting that PEN and CEL would play a role in binding to sRAGE. On the other hand, CEL in BSA-AGEs prepared with $20 \mathrm{mM}$ MGO hardly contributed to binding to sRAGE (Fig. 3A). Although CEL and PEN concertedly contributed to sRAGE binding, anti-CEL Ab and anti-PEN Ab could not fully suppress sRAGE binding to BSAAGEs prepared with $100 \mathrm{mM}$ MGO (Fig. 3C). These observations indicate that other MGO-derived AGEs on lysine, such as methylglyoxal lysine dimer (MOLD) and methylglyoxal derived imidazoline crosslink (MODIC), might be additionally required for the binding of BSA-AGEs prepared with 50 and $100 \mathrm{mM}$ MGO to sRAGE. A further study would be required for testing this possibility when antibodies specific to MOLD and MODIC are available.

In summary, we have proposed a method for screening RAGEbinding AGEs, which includes lysine methylation and a competitive assay using biotin-tagged sRAGE and an AGEsspecific antibody. When this method was applied to BSA-AGEs produced by MGO, CEL and PEN partly contributed to RAGE binding, but AGEs produced on arginine were not largely involved in RAGE binding. Although lysine methylation was an optional step, this might help to eliminate AGEs that were free from RAGE-binding, despite a limited availability of the AGEsspecific antibody.
A

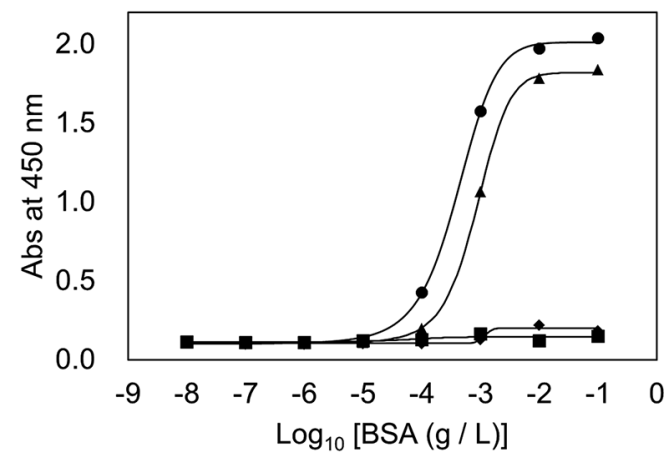

B

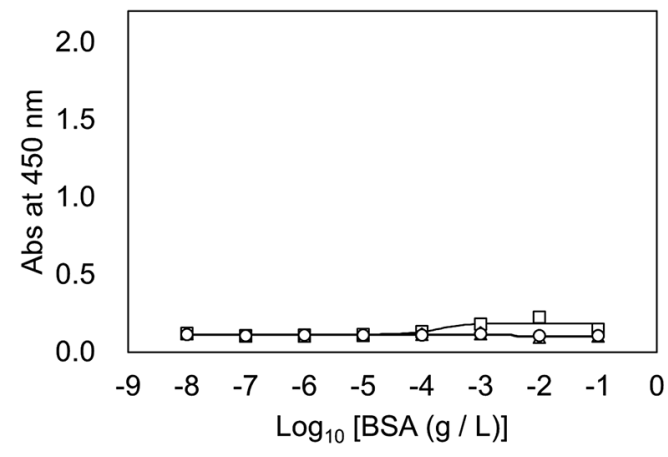

C

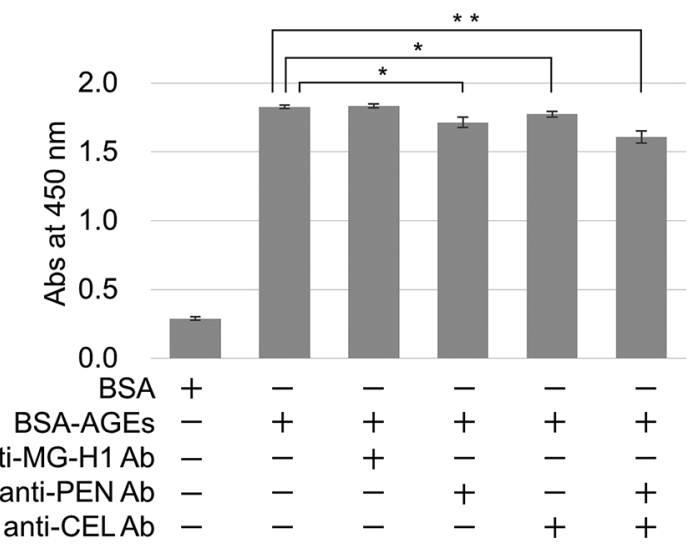

Fig. 3 Binding of sRAGE to AGEs. All sets of profiles of sRAGE binding to BSA-AGEs samples (A) and MeBSA-AGEs samples (B) were fitted with four-parameter logistic model. Symbols in panels (A) and (B) indicate as follows: filled square (BSA); filled diamond (BSAAGEs with $20 \mathrm{mM}$ MGO); filled triangle (BSA-AGEs with $50 \mathrm{mM}$ MGO); filled circle (BSA-AGEs with $100 \mathrm{mM}$ MGO); open square (MeBSA); open diamond (MeBSA-AGEs with $20 \mathrm{mM} \mathrm{MGO);} \mathrm{open}$ triangle (MeBSA-AGEs with $50 \mathrm{mM}$ MGO); open circle (MeBSAAGEs with $100 \mathrm{mM} \mathrm{MGO}$ ). Binding of sRAGE to AGEs in the presence of AGEs-specific antibodies was analyzed (C). The data obtained from three independent experiments were analyzed for any statistical difference using the Student's T-test. Single and double asterisks indicate significant differences $(p<0.05 v s$. control for single asterisk, $p<0.01 v s$. control for a double asterisk).

\section{Acknowledgements}

This work was supported by a Grant-in-Aid for Scientific Research (C), 18K05544, from the Ministry of Education, Science, Sports and Culture, and a grant from the Ministry of Agriculture, Forestry and Fisheries of Japan (Research Project for Sericultural Revolution). 


\section{Supporting Information}

Supporting Information is available free of charge on the Web at http://www.jsac.or.jp/analsci/.

\section{References}

1. S. Arena, A. M. Salzano, G. Renzone, C. D’Ambrosio, and A. Scaloni, Mass Spectrom. Rev., 2014, 33, 49.

2. J. O'Brien, P. A. Morrissey, and J. M. Ames, Crit. Rev. Food Sci. Nutr., 1989, 28, 211.

3. R. Bucala, Z. Makita, G. Vega, S. Grundy, T. Koschinsky, A. Cerami, and H. Vlassara, Proc. Natl. Acad. Sci. U. S. A., 1994, 91, 9441.

4. J. Uribarri, M. D. del Castillo, M. P. de la Maza, R. Filip, A. Gugliucci, C. Luevano-Contreras, M. H. MacíasCervantes, D. H. Markowicz Bastos, A. Medrano, T. Menini, M. Portero-Otin, A. Rojas, G. R. Sampaio, K. Wrobel, K. Wrobel, and M. E. Garay-Sevilla, Adv. Nutr., 2015, 6, 461.

5. M. Koch, S. Chitayat, B. M. Dattilo, A. Schiefner, J. Diez, W. J. Chazin, and G. Fritz, Structure, 2010, 18, 1342.

6. R. Ramasamy, S. F. Yan, and A. M. Schmidt, Amino Acids, 2012, 42, 1151.

7. G. Fritz, Trends Biochem. Sci., 2011, 36, 625.

8. H. Yang, H. Wang, S. S. Chavan, and U. Andersson, Mol.
Med., 2015, 21(Suppl 1), S6.

9. M. O. Chaney, W. B. Stine, T. A. Kokjohn, Y.-M. Kuo, C. Esh, A. Rahman, D. C. Luehrs, A. M. Schmidt, D. Stern, S. Du Yan, and A. E. Roher, Biochim. Biophys. Acta-Mol. Basis Dis., 2005, 1741, 199.

10. S. Yamagishi and T. Matsui, Oxid. Med. Cell. Longev., 2010, 3, 101.

11. J. Xue, V. Rai, D. Singer, S. Chabierski, J. Xie, S. Reverdatto, D. S. Burz, A. M. Schmidt, R. Hoffmann, and A. Shekhtman, Structure, 2011, 19, 722.

12. M. Kumano-Kuramochi, K. Tatematsu, M. OhnishiKameyama, M. Maeda-Yamamoto, T. Kobori, H. Sezutsu, and S. Machida, Sci. Rep., 2017, 7, 356.

13. T. S. Walter, C. Meier, R. Assenberg, K.-F. Au, J. Ren, A. Verma, J. E. Nettleship, R. J. Owens, D. I. Stuart, and J. M. Grimes, Structure, 2006, 14, 1617.

14. T. Usui, K. Shimohira, H. Watanabe, and F. Hayase, Biosci. Biotechnol. Biochem., 2007, 71, 442.

15. A. Lorenzen and S. W. Kennedy, Anal. Biochem., 1993, 214, 346.

16. R. E. Smith and R. MacQuarrie, Anal. Biochem., 1978, 90, 246.

17. N. Ahmed, P. J. Thornalley, J. Dawczynski, S. Franke, J. Strobel, G. Stein, and G. M. Haik, Invest. Ophthalmol. Vis. Sci., 2003, 44, 5287.

18. G. Vistoli, D. De Maddis, A. Cipak, N. Zarkovic, M. Carini, and G. Aldini, Free Radic. Res., 2013, 47, 3. 